\title{
The crystal's in the mail...
}

\author{
Do you need to solve the structure of a complex biological molecule quickly, \\ or require some expert help doing it? No problem. Tracy Smith Schmidt \\ samples the delights of 'mail-in' crystallography.
}

$\mathrm{S}$ ome say that today's biology graduate students are spoilt. Not so long ago, they had to do all their own routine bench work - from sequencing DNA to making transgenic mice. But core facilities at many universities now provide these services reliably and cost-effectively. Budding researchers, freed from this treadmill, can concentrate on more interesting experimental work.

The next generation could be even more pampered. If they need to solve the crystal structure of a protein, they may not have to master every aspect of X-ray crystallography. Instead, biologists might simply mail crystals of the protein to a specialist centre, where expert staff will perform the X-ray analysis and send back the data electronically. Indeed, some pioneering facilities are already offering such 'mail-in' services.

Solving the structure of a protein, or any other complex biological molecule, is a laborious task that used to take years. First, the protein has to be purified from cell culture, before the painstaking process of finding conditions to get it to crystallize from solution. The crystals are then irradiated with X-rays, to see how the beam is scattered by the crystalline array of atoms. From these data, a three-dimensional map is generated that shows the location of each atom within the molecule.

\section{Booking time}

Although the basic steps haven't changed over the years, the time it takes to solve a structure has, dropping to only a few months in many cases. Many technical advances have helped turn this once arcane pursuit into a staple technique of modern biology. In particular, synchrotron facilities - machines in which electrons moving at close to the speed of light are steered by magnetic fields around a huge, ring-shaped accelerator to generate high-intensity X-ray beams - have made many more experiments possible. For example, high-quality data can be collected from tiny crystals that were once deemed unusable.

But booking time to collect data at a synchrotron beamline is still a limiting stepand for crystallography novices, an extremely daunting one. Fortunately, there's now an alternative. Since 2000, the National Synchrotron Light Source at the Brookhaven National Laboratory in Upton, New York, has offered a

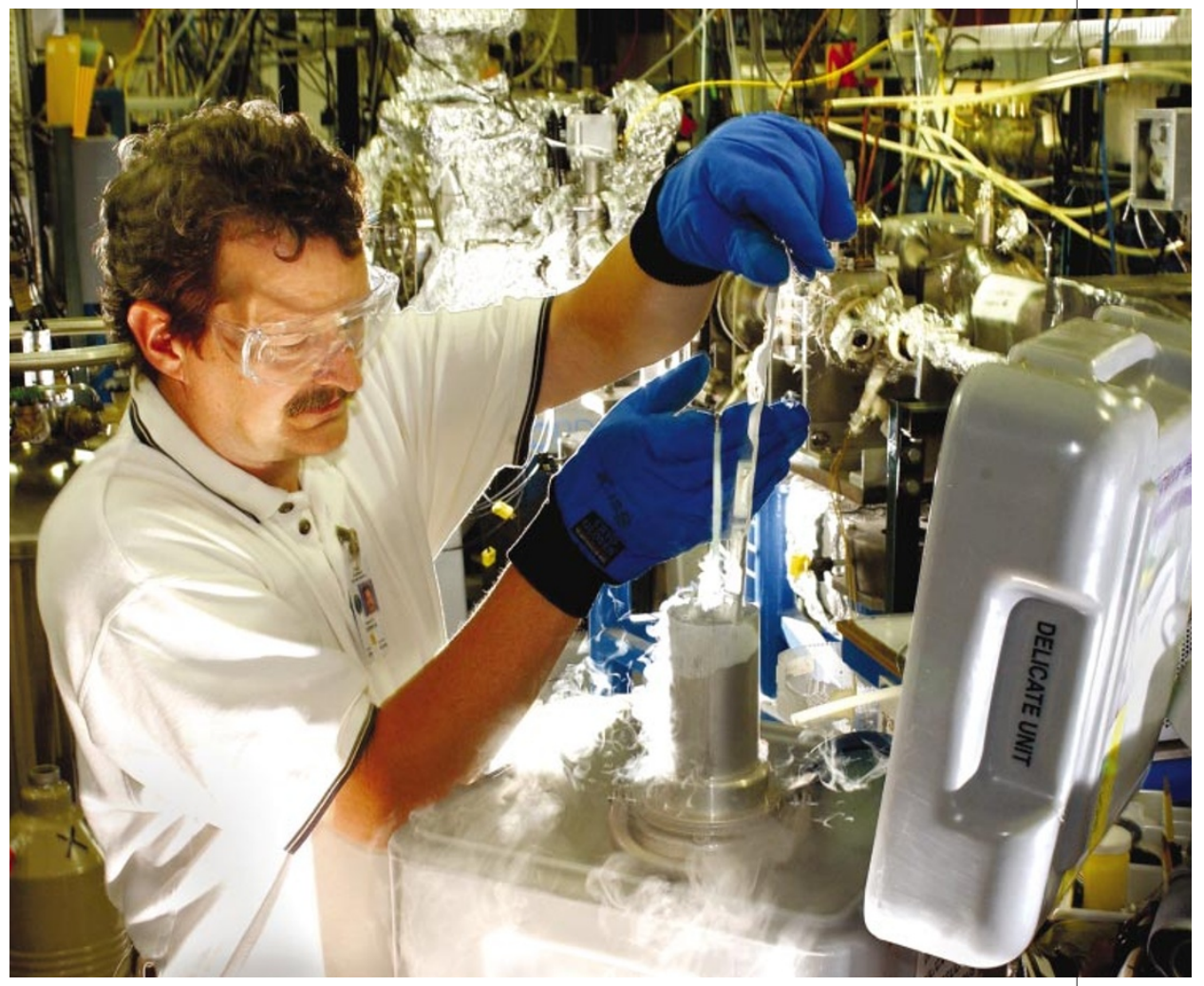

At your service: Howard Robinson examines a sample sent to Brookhaven for structural analysis.

mail-in data-collection service: you supply the crystal and they deliver the data.

Run by Howard Robinson, the Brookhaven programme contributes to more than 50 projects each year, offering its services free to academic scientists. Researchers whose proposals are accepted send in their crystals. Robinson's experienced crew collects the $\mathrm{X}$-ray diffraction data, often making recommendations about how best to perform the experiment. Finally, they forward minimally processed data to the user. Unless everyone agrees to collaborate further, Robinson's group does not calculate the final set of atomic coordinates that allows researchers to display all the twists and turns in the protein.

About half of Brookhaven's applicants have been molecular biologists with little previous crystallography experience. The rest have been established structural biologists who are in a hurry to get something done, and who are more than happy for Robinson's team to help them out.

The high demand for synchrotron time means that crystallographers typically have to apply for a slot on an X-ray beamline months in advance. When they travel to the synchrotron, they must often run their experiments in marathon sessions of 12 to 24 hours. And if they fail to obtain all the data they need, it's back to the end of a long queue. In contrast, Robinson's mail-in service usually delivers results within a few weeks of submitting a proposal.

For non-crystallographers, access to expertise, rather than speed of service, is a major draw. Ziwei Huang, a biochemist at the University of Illinois at UrbanaChampaign, studies chemokines, proteins involved in coordinating the immune response. He had long been interested in pursuing structural work but was unsure how to jump into the field. Over the past couple of years, his team has made the leap, thanks to the Brookhaven mail-in service and to a facility on Huang's home campus that helped his graduate student prepare crystals of the chemokines they wanted to analyse. 


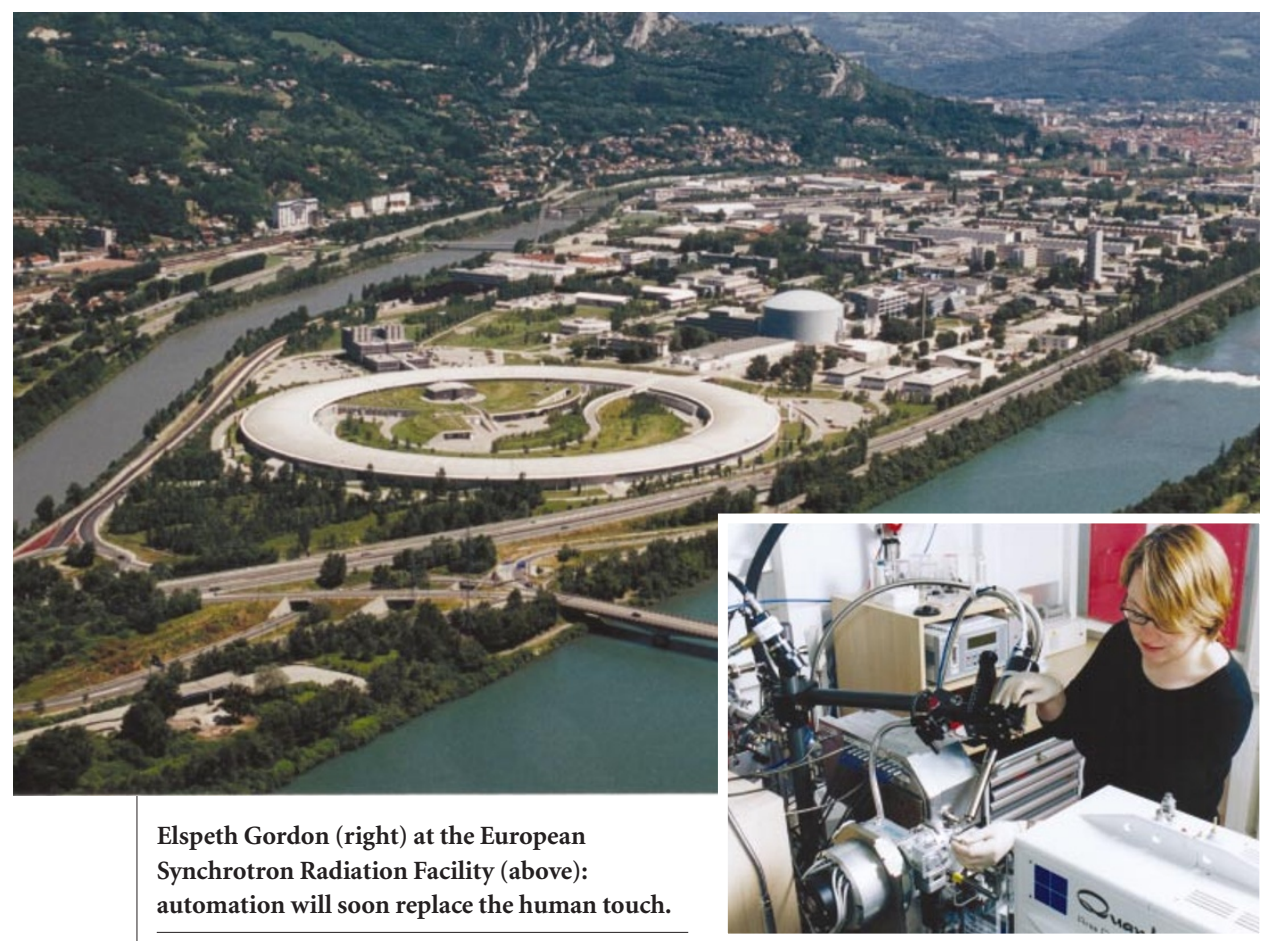

"As I did not have much prior experience in this area, without these services it would have taken us much longer to go in this direction," says Huang. Having been introduced to the world of crystallography, his group now has the confidence to pursue independent structural studies.

But taking advantage of expert knowledge in this way does introduce complications not least the thorny issue of how the experts should be credited for their work. Robinson says that he expects members of his team to be offered co-authorship of papers if they make a "significant contribution" to a project. But there are no hard-and-fast rules. "It's a grey area," says Robinson. "We try to decide the needs of the project, and users have to decide what is a major contribution."

\section{Express service}

The Brookhaven group is not alone in offering mail-in data collection. At the European Synchrotron Radiation Facility (ESRF) in Grenoble, France, Elspeth Gordon and Stéphanie Monaco run an operation called MXpress, which provides a mail-in service for a fee to anyone willing to pay.

For Vincent Mikol, head of structural biology with Aventis Pharma in Paris, MXpress has freed his staff from the travel and unsociable hours associated with working at a synchrotron facility. "We really wanted to avoid spending time, specifically so many nights, at the synchrotron," he says. So a year ago, Aventis entered into an agreement with Gordon and Monaco that has so far provided data on more than 200 biological samples.

Mikol is pleased with the results, but acknowledges that staff unused to the idea of trusting a third party to handle their precious samples had to overcome some mental roadblocks. "At first people were reluctant to send new crystals, but after a while, they saw that the ESRF group was responsive and capable," says Mikol. "We still travel to synchrotrons a bit, but the goal is to have this fade out completely in the next two years."

Trust is an issue for other researchers as well. "If the crystallization were straightfoward and reproducible, we would definitely be keen to try a service such as Brookhaven's," says Brenda Schulman, a structural biologist at St Jude Children's Research Hospital in Memphis, Tennessee. "But using a mail-in service for projects where every crystal is extremely precious could put us both in an awkward situation if something were to happen to the samples or if the data collected were not optimal."

These concerns may lessen within the next few years, as data collection becomes fully automated. Machines, not technicians, will pick up the crystals, mount them on the apparatus, centre them in the X-raybeam, and collect the data. Bar-coding samples should further reduce the scope for human error.

In addition, remote-access software is being developed to allow scientists to observe and manipulate operations at a synchrotron

\section{W e wanted to avoid spending} so many nights at the synchrotron. Vincent Mikol from their own labs. Users will be able to monitor their data as they appear and make judgement calls about how to proceed — for example, to request a move to another crystal if the one in the beam is of poor quality. Once this capability is in place, scientists will be able to stay intimately involved in the execution of their own experiments - they will just be physically removed from the site of data collection. The major issue to overcome is security: how to create firewalls to prevent hackers from gaining access to a user's results and to the beamline equipment.

\section{Keeping in touch}

But even when this technology is available, some researchers may still prefer the handsoff approach of having the synchrotron staff run their experiments. Some scientists raise the question of whether new recruits will suffer in the drive for greater efficiency and miss out on learning the intricacies of crystallography. "We run the risk of not training graduate students at the beamline," says Stephan Ginell, who coordinates the Structural Biology Center User Program at the Advanced Photon Source, a synchrotron at the Argonne National Laboratory in Illinois.

Most structural biologists are confident that a balance can be struck. Ashley Deacon of the Stanford Synchrotron Radiation Laboratory in California, for one, believes that many researchers will want to stay hands-on - which should ensure that graduate students get the training they need. "For the most part, researchers still want to be highly involved," he says. "A lot of human interpretation still goes into it."

Even so, it may eventually become standard for biologists to contract out crystallographic procedures. Already there are companies, such as Rigaku/MSC of The Woodlands, Texas, offering the full gamut of services, from crystallization to structure solving.

As more outsourcing opportunities emerge, the issue of training may become moot. "I used to spend a great deal of time as a graduate student sequencing DNA, but now this is done as an automated service," observes Schulman. "Mastering particular protocols may be less important than learning the process of how to do good science." Still, she notes that researchers who rely solely on others to perform their structural analysis may risk overlooking errors and artefacts in the data.

As this debate continues, the signs are that demand for mail-in crystallography is growing. Those synchrotrons that haven't yet offered the service are getting requests to do so. "I get calls from people demanding it for general users," says Ginell.

Tracy Smith Schmidt is a freelance writer in New York and was formerly editor of Nature Structural Biology. www.px.nsls.bnl.gov www.esrf.fr/Industry/MX/MXpress 